\title{
Phase plates for wave-aberration compensation in the human eye
}

\author{
Rafael Navarro and Esther Moreno-Barriuso \\ Instituto de Optica "Daza de Valdés," Consejo Superior de Investigaciones Cientificas, Serrano 121, 28006 Madrid, Spain
}

Salvador Bará and Teresa Mancebo

Área de Óptica, Facultade de Física, Universidade de Santiago de Compostela, 15706 Santiago de Compostela, Galicia, Spain

Received June 22, 1999

\begin{abstract}
We present a method for manufacturing phase plates to compensate for the wave aberration in the human eye. The wave aberration of the eye is measured in vivo by a new laser ray-tracing method and then compensated for by a phase plate placed in front of the eye. This plate is made from a gray-level single-mask photosculpture in photoresist. Two experiments were carried out, first with an artificial eye and then with a human eye: $80 \%$ compensation for the wave aberration was achieved in both cases. () 2000 Optical Society of America

OCIS codes: $220.0220,330.0330,020.3620$.
\end{abstract}

In $1961 \mathrm{Smirnov}^{1}$ reported measurements of the overall wave aberration in the human eye obtained by means of a spatially resolved subjective refractometer. He suggested that "in principle, it is possible to manufacture a lens compensating the wave aberration of the eye in the complex form of the plates of errors." We have verified Smirnov's suggestion experimentally by manufacturing and testing such special lenses. These lenses consist of phase plates adapted to compensate for optical aberrations in individual eyes. Conventional opthalmic lenses can compensate for second-order aberrations (defocus and astigmatism) only, and only recently was the first successful attempt at overall phase conjugation in the human eye reported. ${ }^{2}$ In that experiment Liang et al. combined a Hartmann-Shack wave-front sensor and a deformable mirror, similar to those used in some astronomical telescopes. This sophisticated system is, however, far from the idea of a special single lens, and it is hard to imagine its working outside the environment of the optical laboratory. Special lenses were made before, not for correction of the overall wavefront distortion but for partial correction of specific types of aberration (spherical, ${ }^{3}$ coma, ${ }^{4}$ etc.).

In this Letter we report what to our knowledge are the first experimental results of manufacture and testing of customized phase plates (special lenses) for the conjugation of the overall monochromatic $(\lambda=$ $543 \mathrm{~nm}$ ) wave aberration in individual eyes. Our results, with $80 \%$ aberration compensation attained in both artificial and human eyes, constitute what we believe is the first successful implementation of Smirnov's suggestion. The phase plate is a light single component, which permits us to build a static open-loop system for phase conjugation.

The complete experiment consists of three stages that we tested independently. The first step is to measure the aberrations in the eye under test, by a laser ray-tracing (LRT) method. ${ }^{5}$ The LRT had been described and validated previously in a series of experiments, including direct comparison with a Hartmann-Shack wave-front sensor. ${ }^{6}$ The wave aberration is then obtained by the standard method based on least-squares fitting of the geometric aberrations to the derivatives of Zernike polynomials. ${ }^{7}$ The next step is to manufacture a phase plate with Zernike coefficients of the same magnitude but opposite sign. To make the plates we applied a gray-level single-mask photosculpture in photoresist technique. ${ }^{8-10}$ After passing optical tests (see below), the plate is placed in front of the eye like a spectacle lens (except that it requires careful alignment with the eye's pupil for maximum compensation). In the third step, we measure the wave aberration again to evaluate the degree of compensation attained, as a global assessment of the whole procedure.

Thus there are two main techniques involved, LRT and photosculpture. The basis of the LRT method is depicted in Fig. 1. A narrow Gaussian laser beam (0.7-mm diameter) is used as a finite approximation of a ray of light. A two-dimensional laser scanner permits the beam to be directed to the desired coordinates at the entrance pupil plane. The aerial image $\mathrm{A}^{\prime}$ of (Gaussian) spot A, formed on the retina by the beam, is collected by a CCD camera, and its centroid coordinates are then computed. By delivering a sequential series of rays coming from the same point object and passing through a previously defined pupil sampling grid, we obtain a spot diagram. In this experiment the grid is hexagonal with $6-\mathrm{mm}$ diameter and 1-mm steps (37-ray set). The effective pupil diameter is roughly $6.7 \mathrm{~mm}$ because of the finite width of the beam. A seventh-order Zernike polynomial expansion, 35 coefficients, close to the number of samples, is used to describe the wave aberration of the eye.

This set of 35 coefficients, which is the output of the measuring stage, is the input for the phase-plate manufacturing process. For this purpose we implemented a gray-level single-mask photosculpture in photoresist technique, which is a reasonably efficient and cheap method for making micro-optics elements. We start with a flat plate that is a cleaned soda lime glass substrate spin coated with Shipley S1828 positive photoresist at $400 \mathrm{rpm}$ during $30 \mathrm{~s}$ and prebaked at $90{ }^{\circ} \mathrm{C}$ for $30 \mathrm{~min}$. In this way we obtain a thin photoresist film that is $\approx 12 \mu \mathrm{m}$ thick (see Fig. 2). This 


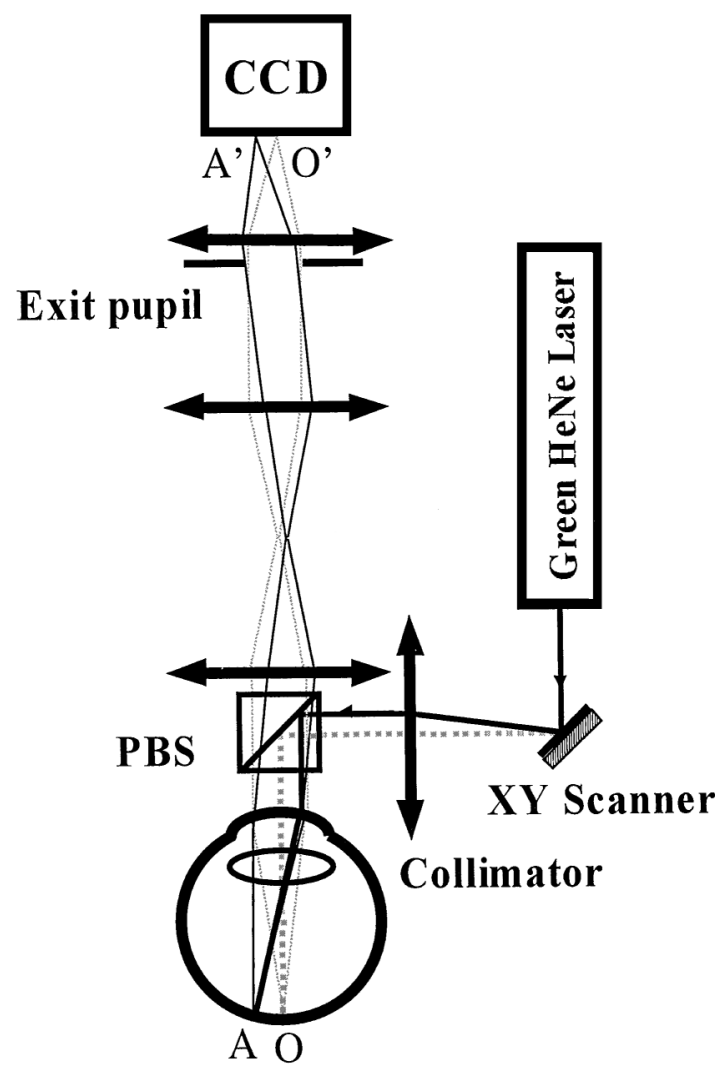

Fig. 1. LRT method: A narrow laser pencil is deflected by a computer-controlled two-dimensional laser scanner. The beam, after passing through a beam splitter and a given point at the pupil plane, forms a small spot of light at the retina. The position offset, $\mathrm{AO}$, between this spot and that formed by the chief ray is the aberration of that ray. A CCD camera records the image of each spot to compute its centroid. PBS, polarization beam splitter.

plate is exposed to ultraviolet radiation (Fig. 2) through a variable-transmittance mask that encodes the desired phase. We fabricated the mask by photoreducing a hard copy of the (negative) pattern obtained with a conventional ink injection printer. After development, the variable-irradiance dose absorbed by the photoactive compound gives rise to a three-dimensional continuous profile of unequal depth. A depth difference $\Delta z$ will produce a phase lag $2 \pi(n-1) \Delta z / \lambda$, where $n$ is the refractive index of the photoresist. By a careful calibration of the entire process, which has shown reasonably good linearity, it is possible to obtain a plate that will produce a phase disturbance that is conjugated (opposite) to the wave aberration of the eye. Hence the resultant phase plate can potentially act as a correcting element. We verify that the phase generated by the plate is close to the nominal desired value by using a Mach-Zehnder interferometer. At the bottom of Fig. 3 we compare the ideal reference (left) and the resultant (right) interferograms measured for the plate used for our subject's eye. We can see that, despite its granular appearance, which is due to manufacturing noise and errors, there is a reasonable overall similarity between the interferogram (phase of the resulting plate) and the nominal design value.
Nevertheless, we measured the wave aberration of the plate (alone) by laser ray tracing. The resultant rms difference between the measured and the nominal wave aberration was $0.25 \mu \mathrm{m}$ in this case (Fig. 3). The resultant plate is placed at a distance of $10 \mathrm{~mm}$ in front of the eye (between the eye and the beam splitter in Fig. 1) and carefully aligned with the optical axis of the system.

The first experiment was carried out with an artificial eye consisting of a high-quality, low-numericalaperture achromatic doublet lens and a white diffusing surface, placed at the focal plane of the lens, acting as an artificial retina. We introduced wave-front distortions into this ideal eye by placing an aberrating plate (soda lime glass deformed by heating) close to the lens. The measured rms wave-front error was $0.79 \mu \mathrm{m}$ initially but decreased to $0.16 \mu \mathrm{m}$ after insertion of the correcting plate, that is, an $80 \%$ compensation.

The results of the second experiment (right eye of subject EM) are shown in Fig. 3. The degree of compensation (compare the two top figures) is again $80 \%$. Such a substantial compensation, which includes third- and higher-order aberrations, confirms that aberrations can be corrected in living human eyes by customized phase plates. This result looks promising, because further improvements of the method are still possible (especially in the manufacture of the plates).

There are several limitations that are inherent in all phase-conjugation methods: Compensation is possible only for a limited visual field and for a given accommodative state. In addition, the alignment becomes critical in the compensation for high-order terms. Our method also has some specific advantages and drawbacks. The main advantages are the moderately low cost and the high spatial resolution. The drawbacks are the specificity of a plate for a single subject or condition (unlike adaptive devices) and the
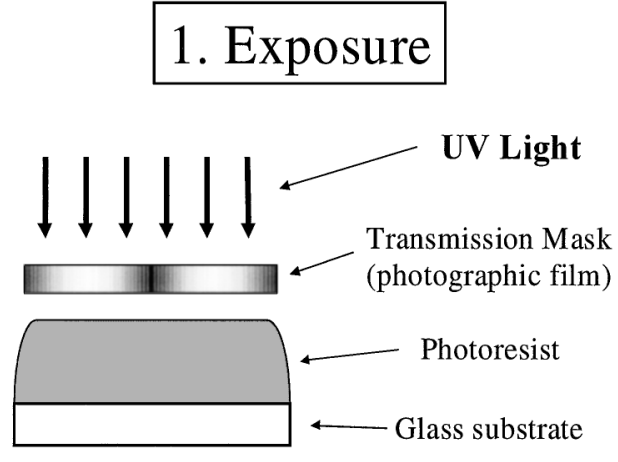

\section{Result (after developing)}

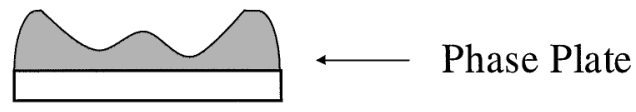

Fig. 2. Manufacture of phase plates. The photoresist deposited upon a glass substrate is exposed to UV radiation through a gray-level transmission mask. After developing, we obtained the desired profile. 

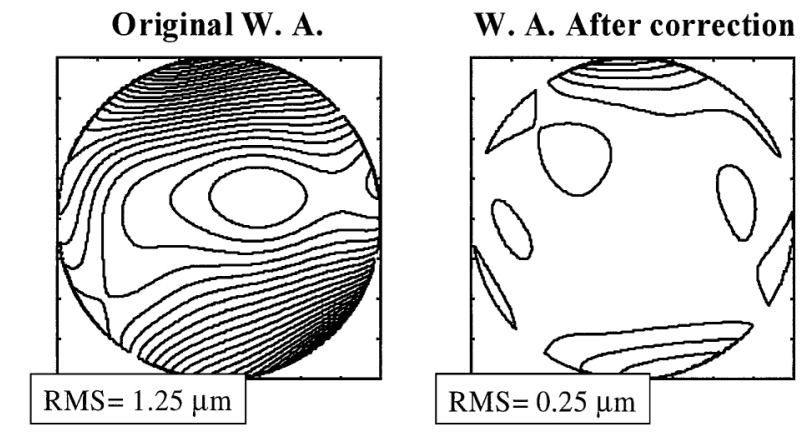

Ideal Interferogram

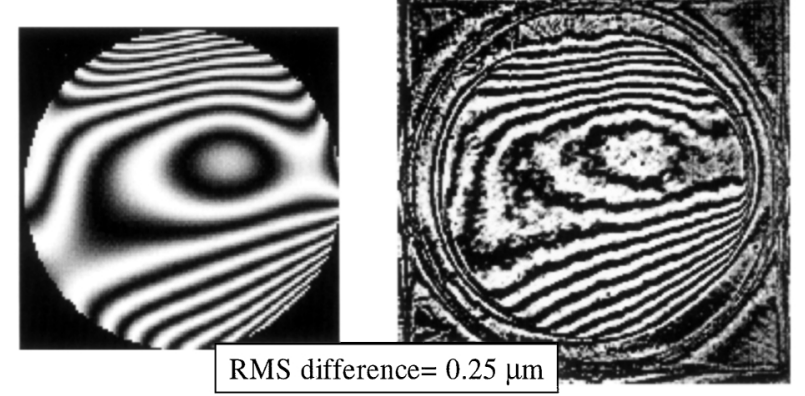

Fig. 3. Top, wave aberration (W. A.) of subject EM (right eye) before (left) and after (right) compensation. The contour-line step is $0.5 \mu \mathrm{m}$. Bottom, ideal (left) and real (right) phases of the plate, measured by a Mach-Zehnder interferometer; the rms difference between the design (ideal) and the manufactured plate is $0.25 \mu \mathrm{m}$.

lower transparency of the photoresist compared with optical glass (although it would be possible to manufacture glass replicas by standard techniques).

For subject EM the rms residual aberration after compensation was the same as the rms difference between the nominal and the measured phases of the plate $(0.25 \mu \mathrm{m})$. This suggests that inaccuracies in the manufacture of the plate were the main limitations on the compensation attainable in this case. Manufacture has two main practical constraints: (1) Accuracy is limited by the minimum structure that we can sculpt with some reliability. (2) The maximum magnitude of aberration that we can compensate for is limited, because as we increase the thickness of the photoresist layer it becomes harder to control the layer's flatness.
Overcoming these two problems has contradictory solutions, but both accuracy and dynamic range are necessary because low-order aberrations in the eye can have high values (several wavelengths), whereas their magnitude tends to decay rapidly with order. ${ }^{11}$ Thus compensating for low (high values) and high (low values) orders simultaneously is difficult. Nevertheless, considering that second-order aberrations can be compensated for with conventional lenses, and that the contribution of higher orders is small in general, it seems more efficient to make plates for correcting a more limited set of aberrations (third, fourth, and possibly fifth orders). In this case, because the required dynamic range will be much lower this subset of aberrations could be compensated for with higher accuracy. In a next step, we intend also to explore the possibility of using an ophthalmic lens (adapted to the eye's prescription) as the substrate upon which to deposit the photoresist to obtain a monolithic single optical element for high-accuracy compensation.

This research was supported by the Comision Interministerial de Ciencia y Tecnología of Spain, grant TIC98-0925-C02. R. Navarro's e-mail address is r.navarro@io.csic.es.

\section{References}

1. M. S. Smirnov, Biofizika 6, 687 (1961) [Byophysics 6, $766(1962)]$.

2. J. Liang, D. R. Williams, and D. T. Miller, J. Opt. Soc. Am. A 14, 2884 (1997).

3. N. Chateau, A. Blanchard, and D. Baude, J. Opt. Soc. Am. A 15, 2589 (1998).

4. N. López, H. C. Howland, B. Howland, N. Charman, and R. Applegate, J. Opt. Soc. Am. A 15, 2563 (1998).

5. R. Navarro and M. A. Losada, Optom. Vision Sci. 74, 540 (1997).

6. R. Navarro and E. Moreno-Barriuso, Opt. Lett. 24, 951 (1999).

7. D. Malacara, Optical Shop Testing, 2nd ed. (Wiley, New York, 1992).

8. H. Andersson, M. Ekberg, S. Hard, S. Jakobson, M. Larsson, and T. Nilsson, Appl. Opt. 29, 4259 (1990).

9. D. R. Purdy, Pure Appl. Opt. 3, 167 (1995).

10. T. J. Suleski and D. C. O'Shea, Appl. Opt. 34, 7507 (1995).

11. J. Liang and D. R. Williams, J. Opt. Soc. Am. A 14, 2873 (1997). 